\title{
La contribution triviale des amateurs sur le Web : quelle efficacité documentaire?
}

Crowd-Sourcing and the Trivial Appropriation of Archives: How Can Their Documentary Efficiency Be Evaluated?

Nathalie Casemajor Loustau

\section{(QpenEdition Journals}

Édition électronique

URL : http://journals.openedition.org/edc/2532

DOI : $10.4000 /$ edc. 2532

ISSN : 2101-0366

\section{Éditeur}

Université Lille-3

Édition imprimée

Date de publication : 1 juin 2011

Pagination : $39-52$

ISBN : 978-2-917562-05-5

ISSN : $1270-6841$

\section{Référence électronique}

Nathalie Casemajor Loustau, « La contribution triviale des amateurs sur le Web : quelle efficacité documentaire? ", Études de communication [En ligne], 36 | 2011, mis en ligne le 01 juin 2013, consulté le 20 avril 2019. URL : http://journals.openedition.org/edc/2532 ; DOI : 10.4000/edc.2532

Ce document a été généré automatiquement le 20 avril 2019

(c) Tous droits réservés 


\title{
La contribution triviale des amateurs sur le Web : quelle efficacité documentaire?
}

\author{
Crowd-Sourcing and the Trivial Appropriation of Archives: How Can Their \\ Documentary Efficiency Be Evaluated?
}

Nathalie Casemajor Loustau

1 En plus de cent ans d'existence, les diverses branches de Bibliothèque et Archives Canada ( $B A C)$ ont collecté plus de 25 millions de photographies. Il aura fallu seulement six ans pour voir le site web Flickr rassembler 5 milliards d'images. Cette brève équation illustre la croissance exponentielle des corpus d'images archivés et publiés sur le Web dans le cadre de pratiques documentaires non-professionnelles. Non seulement les internautes mettent en partage des documents, mais ils contribuent également à classer et à commenter ceux des autres utilisateurs, produisant à la fois des strates de métadonnées et des dynamiques de circulation des images (Casemajor Loustau, 2009a).

2 La spécificité de Flickr dans le champ de la «documentarisation du patrimoine » (Welger Barboza, 2001, 9) tient à la fonction d'interface que ce site a progressivement acquis entre, d'un côté, des institutions de mémoire légitimes, obéissant à des normes professionnelles strictes (encadrement des pratiques de collecte, de conservation, d'inventaire et de catalogage des documents), et de l'autre, un site " collaboratif » fort populaire parmi les amateurs de photographie, siège de pratiques très hétérogènes et faiblement encadrées qui relèvent pour bon nombre d'utilisateurs du «hobby » ou du divertissement. Issu de l'association entre Flickr et la Bibliothèque du Congrès américain, le projet The Commons ${ }^{1}$ témoigne bien de cette interpénétration entre champs documentaires traditionnel et informel, entre la sphère des traces collectives et institutionnalisées, distinguées, reconnues protégées par un statut patrimonial officiel (Davallon, 2006), et la sphère des traces personnelles et ordinaires, témoignages d'une culture du quotidien nouvellement reconstituée en objet d'analyse par les cultural studies (Ben Highmore, 2002). 
3 Tout en s'inspirant du cadre établi par The Commons, d'autres institutions patrimoniales, comme BAC, combinent applications collaboratives sur leur propre site web et création d'un compte sur Flickr. Oscillant entre stratégie de communication (se présenter comme un acteur innovant, répondre aux attentes des usagers en matière de développement des applications dites Web 2.0) et expérimentation de nouveaux dispositifs ${ }^{2}$ documentaires, ce type de projet pose de nombreuses questions concernant l'utilisation des contenus produits par les utilisateurs (User Generated Content).

4 Cet article développe une réflexion sur les plates-formes collaboratives en ligne amorcée lors d'une recherche doctorale, dont la première étape a été présentée à la conférence CIDE 12 (Casemajor Loustau, 2009b). En nous appuyant sur les résultats de ces précédentes contributions, nous questionnerons le lien entre les métadonnées triviales (produites en dehors du champ expert), le renouvellement des figures du public et les critères d'évaluation de cette redocumentarisation " participative ".

Reprenant les conclusions du collectif $\mathrm{R}$. T. Pédauque publiées dans La Redocumentarisation du Monde (2007), J.-M. Salaün propose dans un article intitulé « La redocumentarisation, un défi pour les sciences de l'information" (2007) une synthèse de la notion de redocumentarisation qui éclaire ses enjeux du point de vue du cycle documentaire et de l'organisation d'un rapport au monde ancré dans une perspective "post-moderne ». Il souligne que la numérisation d'un corpus documentaire n'est pas une simple traduction d'un support à l'autre, mais un processus de reconfiguration qui s'opère à travers une nouvelle mise en contexte et de nouvelles formes d'accès. En rendant accessible une collection patrimoniale sur le Web, on contribue donc à sa re-documentarisation, c'est-àdire à l'ajout de nouvelles strates d'information dont les métadonnées constituent le noyau central, modifiant ainsi les conditions de circulation et d'interprétation de cette collection. L'originalité de projets comme The Commons est de proposer aux publics de participer à la redocumentarisation des fonds en apportant des connaissances qui peuvent permettre d'enrichir les métadonnées descriptives des images.

6 Partant du constat établi par R. T. Pédauque sur le lien étroit entre mutations techniques et transformations sociales, nous entendons montrer que l'émergence de tels dispositifs d'indexation collaborative est inséparable d'une évolution dans le domaine des représentations du public, c'est-à-dire leur manière d'interagir avec les documents et la conception de leur rôle au sein des pratiques de production et de transmission des savoirs. Nous questionnerons dans un premier temps les finalités et les modalités de mise en œuvre de ces projets : comment la figure du public actif a-t-elle émergé ? Quelles sont les modalités de contribution proposées aux internautes contributeurs? Dans un second temps, nous interrogerons les modalités d'évaluation de ces projets. Le paradigme de l' efficacité documentaire est-il toujours le plus pertinent pour juger de la production triviale des internautes? Nous discuterons de l'intérêt d'ancrer la perspective dans le paradigme de la démocratie culturelle et dans une poétique de l'ordinaire (de Certeau).

\section{Le rôle actif des publics : une fausse révolution?}

7 En fonction du type d'institution documentaire, de la taille et de la provenance des collections, on observe de plus ou moins grandes lacunes en ce qui concerne la documentarisation des fonds photographiques. Dans le cas de l'inventaire et du catalogage du fonds du studio Notman (fondé à Montréal en 1856), le Musée McCord en 
charge de sa conservation peut s'appuyer sur un registre commercial décrivant de manière très précise l'ensemble des commandes. Mais de tels exemples sont rares, et nombreux sont les cas où les images arrivent accompagnées d'informations textuelles minimales ne permettant pas leur identification.

Malgré ces lacunes documentaires, les institutions patrimoniales peuvent faire le choix de privilégier la mise en accès et de partager ces images numérisées sur leur site web. Quand bien même la notice descriptive d'une image serait complète, solliciter les compétences des internautes peut permettre d'affiner les informations, voire de corriger des erreurs qui auraient pu s'y glisser. «Parce que nos descriptions ne sont pas nécessairement toutes harmonisées [...] », explique la coordinatrice des projets de numérisation à $B A C$, «c'est bien de pouvoir enrichir nos descriptions avec l'information que les gens nous donnent ».

On voit donc comment ces projets collaboratifs s'ancrent dans une représentation des internautes comme public actif, dépassant la simple posture de récepteur pour entrer dans un rôle de producteur de données potentiellement utiles pour l'institution patrimoniale. Mais peut-on vraiment attribuer ce renversement de perspective à l'essor récent des applications 2.0 ? Celles-ci ne sont-elles pas plutôt la manifestation d'une tendance de fonds plus large et plus ancienne?

10 Sur son site web ${ }^{3}, B A C$ encourage les usagers à fournir de l'information sur les images ou plus simplement à partager leur avis : «Si vous (a) reconnaissez un de vos ancêtres sur une image, (b) avez un lien de parenté avec une personne que vous voyez sur une image, ou (c) vous aimez ou n'aimez pas une image, dites-le-nous ». La vague du Web 2.0 a particulièrement mis l'accent sur la figure d'un usager actif et co-responsable de la conception du dispositif de communication. La «culture participative » portée par cette nouvelle génération d'applications en ligne célèbre le déploiement des réseaux sociaux collaboratifs, des communautés d'usagers, du «Web relationnel », du crowsourcing, voire d'une forme d'intelligence collective (Lévy, 1994).

11 Contrairement à une idée répandue, la redéfinition du rôle du public en tant que récepteur "actif» et "productif» ne date pas de l'invention du Web. Dans le cas du champ patrimonial, elle relève d'une tendance à redéfinir le partage des rôles entre publics et institutions selon les principes du dialogue, de l'interaction, et de l'horizontalité des relations. Parfois qualifié de "post-moderne », ce modèle caractérise une posture de l'institution moins tournée vers elle-même, ses collections et ses savoirs, et plus ouverte sur l'espace social, la communauté et les publics, envisagés comme producteurs de sens.

Dès le début des années 1970, la «nouvelle muséologie » a introduit dans le champ patrimonial un schéma de communication favorisant la participation des citoyens, avec notamment la création d'écomusées et de centres d'interprétation. Dans un article consacré aux « musées numérisés », G. Vidal rapporte que :

En 1987, lors de «l'International Workshop on New Museology », l'accent était mis sur «la libération, les progrès et la transformation de la société par la prise de conscience et la participation de la population » [...]. Cette approche place l'usager comme sujet susceptible de devenir expert de par ses pratiques muséales et prend en compte les interactions entre pratiques de musées et d'autres pratiques culturelles (Vidal, 1998, en ligne).

13 Avec l'essor des médias informatisés dans les dispositifs de médiation culturelle, cette nouvelle conception d'un public actif et participant s'est arrimée au paradigme de l'interactivité. Celui-ci caractérise une démarche de participation active de l'utilisateur 
dans le programme de consultation des contenus d'un dispositif de communication. Reprenant l'argument de J. L. Weissberg, J.-T. Julia (2003) souligne comment la notion d'interactivité est souvent confondue avec celle d'un « dialogue » impliquant l'utilisateur d'un système informatique et la machine. Alors que J. L. Weissberg et J.-T. Julia nous invitent à distinguer interactivité (technique) et interaction (humaine), cette confusion a pu favoriser l'arrimage entre une nouvelle conception du public dans le champ patrimonial (interaction, dialogue, horizontalité) et de nouveaux outils de communication informatisée.

14 L'émergence croisée de la figure d'un public actif dans les champs culturel et informationnel ne doit toutefois pas masquer le présupposé dualiste sur lequel repose l'idée d'un public autrefois passif et devenu actif sous l'influence de transformations récentes. Examinant les présupposés de l'imaginaire du Web 2.0, F. Rebillard a reformulé la critique $\mathrm{du}$ "modèle linéaire et éculé » de la transmission d'information (modèle "télégraphique» ou "mathématique ») en rappelant qu'il repose sur une prémisse faussée: «l'asymétrie dialogique entre l'émetteur (auteur) et récepteur (lecteur)» (Rebillard, 2007, 39). Dès les années 1950, les travaux des chercheurs de l'École de Palo Alto (G. Bateson, R. Birdwhistell et P. Watzlavick) ont largement contribué à remettre en question le caractère unidirectionnel et linéaire de la circulation des messages dans les situations de communication. Ils lui ont opposé une conception de la communication comme processus complexe d'élaboration commune d'un sens, auquel participent les interactants. L'usager n'est donc jamais prisonnier d'un rôle purement passif : il est toujours un producteur de sens face aux messages qu'il reçoit.

15 Si ces remarques préliminaires nous amènent à conclure que les dispositifs collaboratifs du Web 2.0 n'instaurent pas un changement radical dans la répartition des compétences et des pouvoirs entre producteurs et récepteurs de l'information, il semble toutefois que la nouvelle génération d'applications en ligne actualise d'une nouvelle manière cette répartition des rôles, contribuant à les institutionnaliser dans des espaces de contribution normés.

\section{Modalités de contribution à la production des métadonnées}

Comment ces évolutions de la représentation des publics se traduisent-elle dans le champ des pratiques de redocumentarisation et de la production des métadonnées? La partie suivante reprend en les résumant les conclusions d'une analyse présentée à la conférence CIDE 12 au sujet des rôles proposés aux usagers dans les dispositifs collaboratifs de Bibliothèque et Archives Canada. Dans un second temps, nous prolongerons l'examen des modalités de contribution amateur en décrivant la manière dont les différents espaces de publication instituent des normes variables de légitimation.

\section{Les rôles proposés aux contributeurs}

Au-delà de la simple interaction avec les contenus numérisés (activation d'un réseau de fonctionnalités et d'hyperliens qui construisent un parcours de consultation des ressources en ligne), nous avons montré que les dispositifs collaboratifs intègrent et 
contribuent à légitimer les figures d'un usager témoin et d'un usager expert (Casemajor, 2009b).

La figure de l'usager témoin caractérise le fait de puiser dans la mémoire personnelle et les souvenirs des usagers pour contribuer à la redocumentarisation d'un fonds. Selon M. Gellereau, «'Témoigner' c'est à la fois attester de la réalité d'un fait, donner corps au souvenir, créer une ressource pour l'avenir par la compréhension des éléments ou susciter des tensions " (Gellereau, 2006, 45). Si la figure de l'usager témoin intègre une dimension d'expertise (au sens où l'usager est porteur d'un savoir tiré de son expérience vécue), la catégorie de l'usager expert recouvre plus largement l'ensemble des connaissances spécialisées dont les internautes peuvent faire bénéficier les institutions patrimoniales afin de contribuer à l'enrichissement des ressources documentaires.

19 Les initiatives de redocumentarisation sur le site Flickr en sont probablement les meilleurs exemples. Faisant le bilan du projet PhotosNormandie, P. Peccatte indique :

L'un des intérêts du projet est bien sûr de faire appel à des spécialistes aux compétences complémentaires. C'est ainsi qu'un participant régulier est un expert sur les diverses unités combattantes de l'époque tandis qu'un autre possède une excellente connaissance de l'aviation durant la Seconde Guerre mondiale. Nous avons aussi obtenu de nombreuses informations précieuses de la part d'un spécialiste des vues aériennes, plusieurs intervenants nous ont aidé sur le Mur de l'Atlantique, et un collectionneur de cartes postales anciennes a identifié de nombreuses localisations (Peccatte, 2008, 6).

Ces divers exemples montrent comment le partage de témoignages personnels ainsi que le partage d'expertise dans les dispositifs collaboratifs de BAC peuvent contribuer à enrichir les métadonnées descriptives des images. La contribution du public est toutefois contrainte par des normes propres aux différents espaces de publication investis par les internautes.

\section{Des espaces de contribution normés}

21 Les conditions de production des UGC varient en fonction du type d'espace éditorial dans lequel se situent les projets collaboratifs. On peut ainsi distinguer les normes de contribution du site officiel de l'institution patrimoniale, où l'activité des usagers tend à être strictement encadrée, et la participation des internautes sur le site de Flickr dont les normes éditoriales sont plus ouvertes et épousent les contours de la pratique amateur.

Sur le site de BAC les modalités de contribution ne sont pas directes: c'est par courriel que les internautes sont invités à transmettre les informations dont ils disposent. Les commentaires font ensuite l'objet d'une relecture systématique et leur publication en ligne est soumise à validation: "Veuillez noter que les commentaires ajoutés aux photographies ne seront pas affichés immédiatement», indique la page de l'exposition virtuelle "Visages de guerre", "ils seront examinés et seront affichés le plus tôt possible ». Ces modalités d'encadrement tiennent à la responsabilité éditoriale directe de $B A C$ vis-à-vis des contenus publiés sur son site (contenus qu'elle accrédite et légitime). "Que fait-on si quelqu'un met en ligne un commentaire contre le gouvernement?", s'inquiète la coordinatrice des projets de numérisation à BAC. Cette contrainte est moins forte sur un site tiers comme Flickr.

23 Le fait de déplacer l'activité collaborative des usagers sur un site autre que celui de l'institution patrimoniale atténue quelque peu la tension entre, d'une part, la volonté 
d'offrir aux internautes des espaces d'expression et, d'autre part, le besoin de contrôler cette expression publique. Dans le cas de Flickr, l'espace éditorial dans lequel les utilisateurs construisent et partagent des formes de savoir n'est plus strictement celui du site gouvernemental officiel, mais un espace clairement identifié comme relevant du Web social et d'une communauté d'utilisateurs avec ses propres règles d'énonciation et de validation de l'information. Les contenus y sont toujours accrédités par BAC et les discussions restent régulées et animées par l'institution, mais le contrôle de l'activité des usagers est moins fort dans le sens où la contribution est directe, informelle et non soumise à un contrôle avant publication.

Derrière cette question du contrôle et de la légitimation de l'information publiée par les utilisateurs se trouve l'enjeu de l'évaluation de la qualité de ces informations. Selon quels critères évaluer la qualité et la pertinence des contenus produits par les utilisateurs ? Nous allons voir comment, dans le cas de BAC, la multiplicité des objectifs visés par ces projets collaboratifs, ainsi que les conditions de leur mise en œuvre, conduisent à relativiser leur portée en termes de redocumentarisation.

\section{Quels critères d'évaluation?}

Expliquant la démarche de son projet sur Flickr, BAC avance plusieurs objectifs. L'un concerne effectivement la redocumentarisation, l'établissement envisageant d'incorporer les informations créées par les utilisateurs dans ses propres bases de données :

Nous voulons explorer l'information fournie à propos des objets que nous partageons en ligne et examiner la façon dont elle peut-être utilisée afin qu'elle devienne éventuellement un élément plus important de ce qui correspond dans notre collection à un objet historique numérique [...]. Il se peut qu'éventuellement, nous envisagions la possibilité d'incorporer cette information dans nos dossiers. Autrement, il se peut que nous décidions de la laisser exclusivement au sein de ces communautés ${ }^{4}$.

Les autres principaux objectifs poursuivis par ce projet illustrent le glissement d'une perspective de démocratisation culturelle (élargissement de l'accès aux fonds numérisés) vers une perspective relevant de la démocratie culturelle, c'est-à-dire habilitant les usagers à construire un lien personnalisé avec les objets culturels (selon les mots de BAC : "parrainer le dialogue ", "augmenter l'interaction" entre usagers et contenus numérisés) $)^{5}$. Si la production de savoir documentaire peut-être évaluée quantitativement par le nombre de notices effectivement améliorées ou corrigées, les critères d'évaluation sont moins évidents lorsqu'il s'agit de juger de formes d'expression triviales manifestant un lien d'attachement aux objets patrimoniaux.

\section{Le paradigme de l'efficacité documentaire}

On peut qualifier de paradigme de l'efficacité documentaire le modèle d'évaluation définissant comme critère principal la capacité de ces dispositifs collaboratifs à fournir de l'information documentaire pertinente et utile en externalisant leur production dans la sphère des usagers. Du point de vue du taux de participation des usagers et du nombre de corrections effectuées dans les bases de données, nous avons montré que les projets collaboratifs mis en œuvre par BAC sont faiblement efficaces. 
28 En ce qui concerne "Visages de guerre ", un sondage de la base de données ${ }^{6}$ reliée à l'exposition virtuelle montre que la participation des utilisateurs est très limitée : sur 210 images, seules 3 ont été commentées. De même, sur le compte Flickr de BAC qui contenait au moment de d'étude 391 images, seuls 5 des 41 commentaires apportaient effectivement une correction des métadonnées ou un élément supplémentaire d'identification. En comparant ces résultats avec ceux d'autres projets similaires (le compte Flickr de la Bibliothèque du Congrès et sur celui du projet PhotosNormandie), nous avons fait apparaitre que cette faible efficacité n'est pas structurelle, mais qu'elle tient aux modalités de mise en œuvre de ce projet par BAC : parmi l'ensemble des contributions proposées, il convient en effet de faire le tri, de vérifier et de réintégrer l'information pertinente sous la forme de métadonnées normalisées (Casemajor Loustau, 2009b).

Ces résultats révèlent que la stratégie d'animation et l'investissement de moyens humains nécessaires dans ce type de projet tendent à être négligés ou sous-estimés. La tentation est grande en effet de déléguer aux outils informatisés (et à leur capacité d'automatisation) le travail de coordination des pratiques de communication qui se nouent autour des fonds documentaires. Mais s'ils n'apportent pas des éléments supplémentaires d'identification réutilisables sur le plan documentaire, la majorité des commentaires postés par les utilisateurs sur le compte Flickr de BAC sont-ils pour autant insignifiants?

\section{Le paradigme de la démocratie culturelle et la créativité triviale}

Le paradigme de l'efficacité documentaire conduit à évaluer les résultats des projets de médiation collaborative sous l'angle exclusif du bénéfice que l'institution culturelle pourrait tirer de l'activité des internautes pour enrichir ses ressources. Or parmi les finalités visées par BAC se trouvent aussi des objectifs relevant du paradigme de la démocratie culturelle. L'examen des relations entre participation démocratique et dispositifs participatifs sur le Web ne peut faire l'économie d'une critique de l'imaginaire d'Internet, au sens où l'interactivité évoque un certain "pouvoir» de l'utilisateur qui manipule le dispositif technique et l'adapte à ses propres usages (Vidal, 1998) ${ }^{7}$. La critique du Web 2.0 met par ailleurs en évidence le fait que la participation dans les dispositifs collaboratifs reste en pratique limitée à une minorité d'internautes (Rebillard, 2007), et que la figure de l'usager actif est au moins autant liée à une nouvelle facette du modèle consumériste qu'à un modèle de démocratie politique :

Il s'agit de déterminer, écrit G. Vidal, si les technologies interactives participent à

l'évolution des relations publics/musées ou bien à une simple diversification des moyens de diffusion culturelle, par des cheminements hypermédiatiques, conduisant vers le renforcement de l'individualisme exprimé via des technologies dites intelligentes parce que potentiellement personnalisantes et valorisé par les comportements consuméristes (Vidal, 2003, 336).

31 Mais l'encouragement de l'expressivité des publics (Allard, 2009) ne se limite pas à une instrumentalisation des principes démocratiques par la sphère marchande. Il peut également favoriser l'appropriation du patrimoine documentaire. Le statut social et symbolique particulier des contenus patrimoniaux (des «sémiophores » selon le mot de K. Pomian, c'est-à-dire des réceptacles de mémoire historique et d'identité culturelle) requiert de considérer aussi les formes d'appropriation sociale (Deprés-Lonnet, 2000) qui se construisent dans le geste de commenter, de tagger et de réorganiser ces données selon le point de vue personnel des utilisateurs. Dès lors, on en vient à considérer aussi 
ces dispositifs de remédiation comme des espaces d'expression d'un lien affectif entre citoyens et contenus patrimoniaux.

Pour intégrer l'angle de l'expérience esthétique et des processus sensibles à l'origine de la création de lien sociaux (Caune, 2006), l'évaluation des formes d'attachement et d'investissement symbolique construites par les usagers doit dépasser la hiérarchie traditionnelle entre d'un côté le domaine de l'expression experte et légitime et d'un autre côté le domaine de l'expression triviale, connectée à la sphère personnelle et à l'intime. Les commentaires postés par les utilisateurs peuvent en effet exprimer un lien affectif avec un lieu représenté à l'écran: "This is my adopted hometown. Lovely old photo!». L'émotion du souvenir personnel se mêle à l'émotion esthétique provoquée par l'image, celle-ci témoignant en même temps d'une histoire collective partagée :

Le temps de l'expérience esthétique est celui où la personne éprouve un temps de plaisir, d'implication et de reconnaissance identitaire. Et c'est dans une relation qui engage les sens que se construit le rapport avec le groupe et le sentiment d'appartenance à une communauté. C'est alors que se réalise la métamorphose du sensible en symbolique et que le phénomène culturel permet la rencontre entre un événement singulier qui concerne la personne et une signification qui fait sens pour tous ceux qui en vivent l'expérience (Caune, 2006, 17-18).

En définitive, la dimension ludique, imaginative et créative de ces pratiques expressives vient questionner la normativité de notre rapport aux archives. Dans ces espaces d'énonciation ouverts sur la pluralité des compétences d'expression, l'émotion et l'intime côtoient la banalité et la médiocrité, traçant les contours d'une « poétique de l'ordinaire » (de Certeau, 1990).

\section{BIBLIOGRAPHIE}

Allard, L., (2009), Remix Culture : l'âge des cultures expressives et des publics remixeurs, Actes du colloque Pratiques Numériques des Jeunes, CSI, Ministère de la Culture et de la Communication.

Barthes, R., (1980), La chambre claire. Note sur la photographie, Paris, Cahiers du cinéma, 192 p.

Casemajor Loustau, N., (2009a), Diffuser les collections photographiques sur le Web : de nouvelles pratiques de médiation? Étude des formes et stratégies de communication du patrimoine photographique en ligne, Thèse de doctorat en communication, UQAM et Université Charles-de-Gaulle - Lille 3, $445 \mathrm{p}$.

Casemajor Loustau, N., (2009b), « Patrimoine photographique et dispositifs collaboratifs en ligne », dans K. Zreik (dir.), Patrimoines 3.0, Actes du colloque CIDE 12, Montréal : Europia Productions, 2009, pp. 43-57.

Caune, J., (2006), La démocratisation culturelle : une médiation à bout de souffle, Saint-Martin d'Hères, PUG, $205 \mathrm{p}$.

Certeau (de), M., (1990 [1980]), L'invention du quotidien. Tome 1 Arts de faire, Paris, Gallimard, 349 p. 
Davallon, J., (2006), Le don du patrimoine : une approche communicationnelle de la patrimonialisation, Paris, Lavoisier - Hermès Science, $222 \mathrm{p}$.

Després-Lonnet, M., (2000), Contribution à la conception d'interfaces de consultation de bases de données iconographiques, Thèse de doctorat en Sciences de l'information et de la communication, Université Charles-de-Gaulle - Lille 3, 430 p.

Gellereau, M., (2006), Témoigner, mises en scène, mises en textes. Présentation du dossier, in Communication et langages, 149, pp. 45-48.

Halbwachs, M., (1994 [1925]), Les Cadres sociaux de la mémoire, Paris, Albin Michel, viii + 367 p.

Highmore, B., (2002), Everyday life and cultural theory : an introduction, Londres et NY, Routledge, $200 \mathrm{p}$.

Julia, J.-T., (2003), Interactivité, modes d'emploi. Réflexions préliminaires à la notion de document interactif, in Documentaliste. Sciences de l'information, 40, 3, pp. 204-212.

Lévy, P., (1994), L'intelligence collective. Pour une anthropologie du cyberespace, Paris, La Découverte, $245 \mathrm{p}$.

Peccatte, P., (2008), Une plate-forme sociale pour la redocumentarisation d'un fonds iconographique, Actes de la deuxième conférence Document numérique et Société, Paris, ADBS Éditions, pp. 373-389.

Pédauque, R. T., (2007), La redocumentarisation du monde, Toulouse, Cépaduès éditions, 213 p.

Pomian, K., (1987), Collectionneurs, amateurs et curieux. Paris, Venise : XVI ${ }^{e}$-XVIII ${ }^{e}$ siècle, Paris, Gallimard, 367 p.

Rebillard, F., (2007), Le Web 2.0 en perspective : une analyse socio-économique de l'Internet, Paris, L'Harmattan, $158 \mathrm{p}$.

Salaün, J.-M., (2007), La redocumentarisation, un défi pour les sciences de l'information, in Études de communication, 30,1 , pp. 13-23.

Vidal, G., (1998), Vers les musées numérisés : de la visite à la navigation, COMMposite, 98, 1 : http:// commposite.org/98.1/articles/vidal.htm, date de la dernière visite : 16 mars 2009.

Welger-Barboza, C., (2001), Le patrimoine à l'ère du document numérique, Du musée virtuel au musée médiathèque, Paris, L'Harmattan, $313 \mathrm{p}$.

\section{NOTES}

1. Lancé en janvier 2008, le projet The Commons (Les organismes publics) vise à encourager les établissements publics à ouvrir un compte sur Flickr pour partager des images issues de leurs collections patrimoniales. Quarante-six institutions avaient rejoint l'initiative en octobre 2010.

2. La notion de dispositif est employée au sens d'un agencement matériel structuré par un système de représentations sociales (par exemple l'anticipation de figures du public) et organisé dans le but d'atteindre un objectif (la transmission patrimoniale dans les cas traités ici).

3. URL : <http://www.collectionscanada.gc.ca/index-f.html>.

4. Extrait du site Internet de Bibliothèque et Archives Canada.

5. Ibid.

6. Consultation des trois premières photographies affichées pour chaque photographe recensé dans la base, soit 210 images sur 2500 . 
7. Voir aussi S. Proulx et M. Sénécal, «L'interactivité technique, simulacre d'interaction sociale et de démocratie ?», Technologies de l'information et société, 1995, 7, 2, pp. 239-255.

\section{RÉSUMÉS}

À travers l'exemple des dispositifs collaboratifs en ligne proposés par Bibliothèque et Archives Canada, cet article examine les conditions de production et de réutilisation des Users Generated Contents dans un contexte patrimonial. Quels sont les rôles proposés aux internautes contributeurs? Quelles normes encadrent les différents espaces de contribution proposés par BAC? Comment évaluer ces projets? Nous montrerons comment le paradigme de l'efficacité documentaire n'est pas toujours le plus pertinent pour juger de la production triviale des internautes, et que les perspectives de la démocratie culturelle et de la poétique de l'ordinaire permettent de jeter un nouveau regard sur ces contenus.

This article analyzes how user generated content is produced and reused in the field of cultural heritage, more precisely through the case of crowdsourcing projects developed by Library and Archive Canada on its website. What roles are contributors invited to endorse? What are the norms framing user contribution in the various media spaces created by LAC ? How to evaluate these projects? We'll see that the paradigm of documentary efficiency is not always the most relevant to assess the triviality of user generated content. Rooting the perspective in cultural democracy and the poetics of everyday life enables us to cast a new light on this informal content.

\section{INDEX}

Keywords : crowdsourcing, contribution, heritage, redocumentarization, triviality, evaluation, user, documentary effectiveness

Mots-clés : dispositifs collaboratifs, contribution, patrimoine, redocumentarisation, trivialité, évaluation, usager, efficacité documentaire

\section{AUTEUR}

\section{NATHALIE CASEMAJOR LOUSTAU}

GERiiCO - Université Lille 3

GRICIS - Université du Québec à Montréal

Nathalie Casemajor Loustau est docteure en communication, membre associée au laboratoire GERiiCO (Université Lille 3) et au GRICIS (Université du Québec à Montréal). Ses travaux portent sur la médiation culturelle dans le champ du patrimoine (en particulier photographique) et des applications Web. Elle collabore également aux travaux du groupe Vie urbaine et médialité dans la culture montréalaise (Media@McGill). Adresse électronique :

casemajor_loustau.nathalie@uqam.ca. 